\title{
Do You Know What Your Scribe Did Last Spring? The Impact of COVID-19 on Medical Scribe Workflow
}

\author{
Jeffrey A. Gold ${ }^{1,2}$ James Becton ${ }^{2}$ Joan S. Ash ${ }^{2}$ Sky Corby ${ }^{1}$ Vishnu Mohan ${ }^{2}$ \\ ${ }^{1}$ Division of Pulmonary and Critical Care Medicine, Oregon Health and \\ Science University, Portland, Oregon, United States \\ 2 Department of Medical Informatics and Clinical Epidemiology, Oregon \\ Health and Science University, Portland, Oregon, United States \\ Address for correspondence Jeffrey A. Gold, MD, Division of \\ Pulmonary and Critical Care Medicine, Oregon Health and Science \\ University, 3181 SW Sam Jackson Park RD, Portland, OR 97239, \\ United States (e-mail: Goldje@ohsu.edu).
}

Appl Clin Inform 2020;11:807-811.

\begin{abstract}
Keywords

- medical scribe

- COVID-19

- electronic health record

- telemedicine

- virtual care

Objective To understand the impact of the shift to virtual medicine induced by coronavirus disease 2019 (COVID-19) has had on the workflow of medical scribes.

Design This is a prospective observational survey-based study.

Setting This study was conducted at academic medical center in the United States. Participants Seventy-four scribes working in ambulatory practices within an academic medical center.

Interventions All medical scribes received a survey assessing their workflow since beginning of COVID-19 restrictions.

Primary and Secondary Outcomes To assess the current workflow of medical scribes since transition to virtual care. Secondary outcomes are to assess the equipment used and location of their new workflow.

Results Fifty-seven scribes completed the survey. Overall $42 \%$ of scribes have transitioned to remote scribing with $97 \%$ serving as remote scribes for remote visits. This workflow is conducted at home and with personal equipment. Of those not working as scribes, $46 \%$ serve in preclinic support, with a wide range of EHR-related activities being reported. The remaining scribes have been either redeployed or furloughed.

Conclusion The rapid transition to virtual care brought about by COVID-19 has resulted in a dramatic shift in scribe workflow with the adoption of a previously unreported workflow of remote scribing for virtual care. Additional work is now needed to ensure these new workflows are safe and effective and that scribes are trained to work in this new paradigm.
\end{abstract}

\section{Background and Significance}

Medical scribes have rapidly become an integral component of U.S. health care delivery, with nearly $20 \%$ of U.S. physicians employing them. ${ }^{1-3}$ Scribes act as intermediaries between physicians and electronic health records (EHRs) by aiding primarily in documentation, but several reports suggest that they may have additional EHR-related responsibilities including data entry and information gathering. ${ }^{4}$
Concern has been expressed that this functional creep could lead to unintended patient safety consequences, a fact exaggerated by the lack of standards for training and assessing scribes. ${ }^{3,5}$ While studies suggest scribe generated notes have a higher degree of readability than physician notes, ${ }^{6}$ it is less clear that traditional methods to evaluate note quality for physician notes can be utilized for scribes, and in one study using simulated encounters, there were issues with overall note accuracy and a significant received

August 13, 2020

accepted after revision

October 21, 2020 (c) 2020 Georg Thieme Verlag KG Rüdigerstraße 14, 70469 Stuttgart, Germany
DOI https://doi.org/

10.1055/s-0040-1721396. ISSN 1869-0327. 
interscribe variability in the data elements recorded in their individual notes. ${ }^{7,8}$

With the outbreak of the novel coronavirus disease 2019 (COVID-19), there has been a rapid transition to virtual care delivery, telecommuting, and redeploying personnel to meet evolving needs. ${ }^{9}$ This raises the concern that when providers are still employing scribes, the scribes have been thrust into new clinical care delivery models with little training. Specifically, changes in workflows consequent to redeploying scribes have not yet been described in the literature. This study describes the impact of the workflow changes induced by COVID-19 on scribe workflows within a single institution, with close attention to their redeployment.

\section{Methods}

We performed this study at Oregon Health and Science University, an academic medical center with 7 physical facilities, with Epic Systems (Madison, WI) being the sole EHR used. The scribe program conducts all of its own recruitment and internalizes its own training. Scribes are deployed to specific practices based on provider request and predominantly work in ambulatory practices. Please see -Table 1 for demographics and - Supplementary Table S1 (available in the online version) for a list of all the clinics staffed by scribes. Pre-COVID-19 scribes worked predominantly in-person, with a few doing pre-charting from home utilizing an array of different hardware solutions including personal laptops, clinic provided ones and in room desktop computers, depending on individual clinic finances and inroom ergonomics. Detailed data on this, however, was not available pre-COVID-19. For remote access, all employees access the EHR via a secure Citrix gateway.

With the onset of COVID-19 and transition to virtual visits, the OHSU Scribe Program embarked on a quality improvement protocol to assess how current scribes were being redeployed by providers in the new paradigm. The study team developed a survey in conjunction with manager of the Scribe Program (J.B.). Questions were based on both institutional experience and the results of a series of grant-funded site visits conducted by our study team on scribe utilization in different settings. ${ }^{5}$ The survey instrument can be found in the - Supplementary Material (available in the online version). The survey was built and deployed by institutional email in Smartsheet to all scribes currently employed. To maximize the response rate, participants were reminded twice in 2-week intervals to complete the survey. Participants received no compensation for completion. The focus of this first survey was to assess the impact COVID-19 has had on their workflows, roles, and practice locations. Based on the results of this first survey, we discovered that for scribes who were still functioning to directly support provider visits had been redeployed into either a virtual scribing role or a role simply pre-charting within the EHR. To better understand sociotechnical factors in both of these roles, we embedded additional questions into the original survey to further clarify roles; one set for those who stated they participated in virtual scribing and the other for those who stated they were performing previsit EHR-related activities. These two additional instruments were created and distributed in similar fashion to the initial survey, and distributed to all participants who responded to working in either of these two new roles.

All data were analyzed with descriptive statistics using both Microsoft Excel and GraphPad Prism. Differences between groups were determined by ANOVA using GraphPad Prism. All figures were created in GraphPad Prism.

\section{Results}

Overall 74 scribes were surveyed, of which 57 (74\%) completed the initial survey. Demographics of respondents and nonrespondents can be found in -Table 1, with no significant difference between the groups and 34/36 (94\%) practice locations represented (- Supplementary Table S1 (available in the online version)]. Overall, after the onset of COVID-19 and the subsequent virtualization of their clinical practices, only $12 \%$ of respondents continued to work as in-office scribes. Conversely, $42 \%$ worked as remote scribes, with the majority supporting either virtual (video) or telephone visits. Forty-six percent of scribes transitioned from scribing during the clinical encounter to previsit activities, such as charting in the EHR prior to visits. The remaining scribes were either furloughed or redeployed into nondocumentation duties with the majority providing telephone support for information technology, specifically focused around the EHR ( Fig. 1). With this change in the care delivery model, $40 \%$ of scribes report that they needed to learn new EHR skills in order perform their new activities.

A secondary survey to further understand remote scribing was sent to 22 participants, with $18 / 22$ (82\%) completing the survey. Of the remote scribes, $95 \%$ were connected to their providers by audio only (tele-scribing). The majority performed their scribing at home, often using their personal equipment ( - Fig. $2 \mathrm{~A}, \mathrm{~B}$ ). To better understand the pre-charting activities, the survey was redeployed with additional

Table 1 Demographics of respondents, nonrespondent, and total scribe population

\begin{tabular}{|l|l|l|l|}
\hline & Respondents $(\boldsymbol{n}=\mathbf{5 7})$ & Nonrespondent $(\boldsymbol{n}=\mathbf{1 7})$ & Total $(\boldsymbol{n}=\mathbf{7 4 )}$ \\
\hline Female & $42(73.6 \%)$ & $9(52.9 \%)$ & $51(68.9 \%)$ \\
\hline Age $(\mathrm{y})$ & 25.2 & 25.9 & 25.2 \\
\hline Years employed & 1.82 & 1.86 & 1.37 \\
\hline Ambulatory scribe & $56(98.2 \%)$ & $17(100 \%)$ & $73(98.6 \%)$ \\
\hline
\end{tabular}




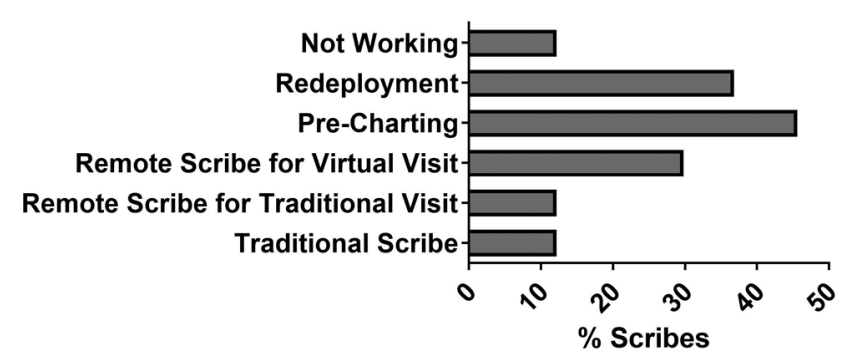

Fig. 1 Redistribution of scribe activity post COVID-19. Medical scribes $(n=57)$ were surveyed for their current workflow post-COVID19. The percent of scribes reporting working in any of the specific activities. Multiple scribes reported working in multiple workflows/roles.

questions surrounding the details of pre-charting activities was sent to the 26 subjects who reported working in this role with $21 / 26$ (81\%) completing the survey. For pre-charting activities, scribes reported a wide variance in duties. While some activities, like chart review and loading note templates, continued to be done the same way as pre-COVID-19; others represented new activities, such as order entry, preliminary medication reconciliation (meaning initial documentation of medication usage), alteration of problem lists, and diagnosis entry (-Fig. 2B). Of greater interest, while almost all were performing chart review (95\%), a variety of other pre-charting activities reported, with none of these activities adopted by $100 \%$ of the scribes and the majority by less than $50 \%$. For the scribes who were redeployed to nonvisit-related activities, $100 \%$ described being assigned to a combination of four new (to them) activities: working in the virtual help desk, being part of a central labor pool, check point staffing, and chart abstraction. Finally, there was no association between the type of redeployment and activities performed with length of time working within the organization.

\section{Discussion}

This study is, to our knowledge, the first to describe the impact the COVID-19 pandemic had on a medical scribe workforce. Our results suggest that scribes are being redeployed and utilized in ways not previously described. While our remote scribing workflows were developed with telehealth and provided to all clinical partners (clinical managers/providers) and scribes alike, the results of this survey with the reported variability highlights the potential for new unanticipated challenges which will be relevant for all organizations.

Scribes in our organization and elsewhere are usually premedical or other pre-professional students with no formal health care training, and they are not licensed career health care professionals. Prior to COVID-19, there were concerns about the risks of scribes going beyond their documentation duties. ${ }^{3,5}$ With workflow changes postCOVID expanding their duties and limiting their interactions with providers to audio only, this rapid transition might result in additional unintended safety consequences.

One area of concern is the transition to remote scribing. While the use of remote scribes has been described previously, the sudden shift to virtual/tele visits due to COVID-19 has now juxtaposed remote scribing with remote patient visits in a way that patient, provider, and scribe are all in different physical locations. ${ }^{5,10,11}$ Therefore, several potential patient safetyrelated concerns arise related to the quality of documentation due to the lack of visual cues as was described in a recent evaluation of multiple scribe models. ${ }^{5}$ Prior studies suggest deterioration with note veracity, which is potentially further confounded by these factors. ${ }^{7}$ This concern is also exacerbated by the observation that the supervision of scribes is varied and unstructured. ${ }^{12}$ This might be exaggerated by physical separation of scribes from their providers, reducing the opportunity for active collaboration and communication. Further, this remote scribing is predominantly performed by scribes who are home and using their personal computer hardware. This use of personal equipment in nonsecure locations raises potential security concerns. There is the potential for data breaches within the institution's clinical information system. Such vulnerabilities were not present prior to scribe redeployment due to COVID-19 and will require organizations to institute set guidelines for this new workflow. ${ }^{13}$

For scribes still involved in patient charting, we observed a wide range of self-reported EHR activities. While many of these
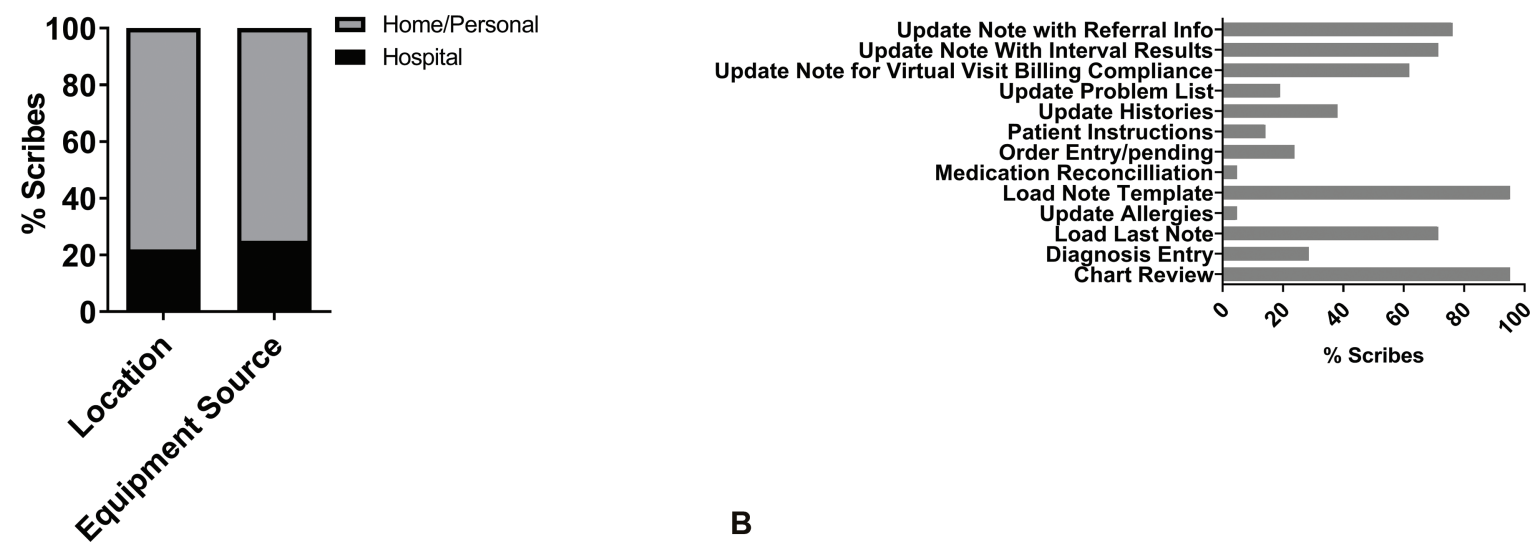

Fig. 2 Survey of scribes redeployed to remote scribing or pre-charting support. (A) Remote scribes were surveyed for the location of their remote scribing and the equipment used. (B) Scribes functioning in pre-charting activities $(n=21)$ were surveyed for the specific EHR tasks they performed. Note, all scribes reported multiple different EHR tasks and data represent the total number of scribes reporting performing each specific activity. 
are well within the domain of scribe activities defined by Joint Commission and others, ${ }^{14}$ a significant number represent scope creep over what is considered the "traditional" role for scribes, including functions such as preliminary medication reconciliation. Of greater interest is the wide variance between scribes in these activities, suggesting this is provider, as opposed to organization driven. When expansion of scope is combined with the lack of standardization in scribe supervision, it has the potential to represent an additional potential safety concern and highlights the importance of a strong and standardized organization culture. Unfortunately, the paucity of national and state standards for scribe-related duties means that the onus will be on individual organizations to set appropriate limits on scope creep and devise their own methods to assess and mitigate it as described in this manuscript and in another recent study. ${ }^{13}$

Finally, the observation that several scribes have either been completely furloughed or redeployed into nonscribe activities within the organization is also of great interest. This means that some providers who were used to having scribes no longer had them available. Of those scribes still employed, all were doing a combination of EHR and non-EHR related tasks (e.g., chart abstraction and checkpoint staffing). The changes in this group's duties as well as those of scribes completely furloughed imply that nearly $50 \%$ of scribes were not functioning in direct provider support. This raises the concern for significant negative impact on providers given the number of studies, which highlight the positive impact scribes can have on job satisfaction. 6,15

This study has some limitations. Most notably, this is a single center study and the organization has developed and deployed its own internal scribe program, as opposed to the more common model of using external scribe staffing agencies. Second, we have no information on the impact of these new changes on provider workflow. The majority of providers utilize scribes because they believe scribes efficiently and effectively use the EHR, thus saving providers' time. What has not been assessed to date is the impact of abruptly removing this assistance for those providers who have become dependent upon scribes to document clinical encounters for them. This raises the potential that the loss of scribes has not only unmasked provider skill atrophy within the EHR, but has also introduced other unintended consequences pursuant to layering new workflows upon new workflows for virtual care. We are currently investigating the impact of this new paradigm since it is not well understood or reported in the literature.

Another limitation is that all of the information obtained on scribe workflow was self-reported. We have no objective assessment of new provider/scribe workflows nor do we have the ability to assess the effectiveness of scribes in this new workflow. With providers delivering virtual care from both their homes as well as work locations, we are also aware that this poses limitations on the generalizability of our findings beyond our institution (a tertiary-care academic medical center located in a major metropolitan area within the United States). Finally, we have no information on the impact virtual scribing has on patient experience and perception, and indeed, this is a topic beyond the scope of the current study.
In conclusion, our study demonstrates, for the first time, the impact COVID-19 had on medical scribes. Given the transitory nature of this workforce and variance in supervision preCOVID-19, it is essential that organizations assess how their scribes have been redeployed, ensure they are properly trained for these new activities, recalibrate the scope of scribe activities to meet the needs of the new paradigm brought on by COVID-19, determine the impacts these redeployments have on the provider, and ensure that infrastructure and training facilitate virtual multi-party collaboration.

\section{Clinical Relevance Statement}

Medical scribes have been rapidly adopted to serve as an intermediary between the provider and the EHR. With the rapid transition to virtual care, the entire paradigm for provider-patient communication is altered, and thus, the role and function of scribes will as well. This study highlights these new challenges and the potential negative unintended consequences if these factors are not prospectively addressed and evaluated by organization and providers.

\section{Multiple Choice Questions}

1. All the statements regarding scribe use are correct except a. Scribes act as intermediaries between physicians and electronic health records (EHRs) by aiding primarily in documentation.

b. Scribes may have additional EHR-related responsibilities including data entry and information gathering.

c. With the outbreak of the novel coronavirus disease 2019 (COVID-19), changes in workflows consequent to redeploying scribes have been well described.

d. Functional scope creep for the scribe role could lead to unintended patient safety consequences, a fact exaggerated by the lack of standards for training and assessing scribes.

Correct Answer: The correct answer is option c. With the outbreak of the novel coronavirus disease in 2019 (COVID19), there has been a rapid transition to virtual care delivery, telecommuting, and redeploying personnel to meet evolving needs. This raises the concern that when providers are still employing scribes, the scribes have been thrust into new clinical care delivery models with little training. Specifically, changes in workflows consequent to redeploying scribes have not been well described.

2. New roles of scribes after redeployment due to COVID-19 included the following except

a. As remote scribes supporting either virtual (video) or telephone visits.

b. Pre-visit activities, such as charting in the EHR prior to visits.

c. Were furloughed.

d. Engaged in one-on-one interviews with patients to establish the history during the visit. 
Correct Answer: The correct answer is option d. Scribes are not allowed to engage in independent practice of clinical care. The authors reported that the majority of scribes who transitioned to assisting physicians provide virtual care assumed a supporting role in either virtual (video) or telephone visits, typically connected by audio only. A significant percent of scribes transitioned from scribing during the clinical encounter to pre-visit activities, such as charting in the EHR prior to visits. While some activities-such as chart review and loading note templates-continued to be done the same way as pre-COVID-19, others represented new activities such as order entry, medication reconciliation, alteration of problem lists, and diagnosis entry

3. What percent of scribes, according to the authors, reported that they needed to learn new EHR skills in order perform their new activities?
a. $10 \%$
b. $40 \%$
c. $70 \%$
d. $100 \%$

Correct Answer: The correct answer is option b. Overall $40 \%$ of scribes reported that they needed to learn new EHR skills in order perform their new activities. This suggests the need to involve scribes in EHR training, in addition to providing maximal support to providers, as organizations transition to the delivery of virtual care expedited by the onset of COVID-19.

4. One finding of the study was that scribes who transitioned to virtual care delivery typically performed their scribing from home, often using their personal equipment (computers and monitors etc.). Which of the following represents the greatest risk to the health care organization utilizing this model?

a. The patient privacy and data security risk associated with using personal computer equipment.

b. The increased risk of on-the-job injuries to scribes subsequent to working in unmonitored environments.

c. The risk of increased turnover of scribes because of their dissatisfaction with their job roles.

d. There are no risks to the organization associated with this model.

Correct Answer: The correct answer is option a. The use of personal equipment in nonsecure locations raises potential security concerns, paramount of which is the potential for data breaches within the institution's clinical information system as a consequence of using unsecured/ unapproved hardware and internet access/communication protocols.

\section{Protection of Human and Animal Subjects}

All work was approved by the institution's institutional review board. The study was deemed minimal risk and therefore granted a waiver of informed consent.

\section{Funding}

This study received its financial support from U.S. Department of Health and Human Services, Agency for Healthcare Research and Quality, R01HS025141.

\section{Conflict of Interest}

None declared.

\section{References}

1 Bossen C, Chen Y, Pine KH. The emergence of new data work occupations in healthcare: The case of medical scribes. Int J Med Inform 2019;123:76-83

2 Martineau M, Brookstone A, Stringham T, Hodgkins M. Physicians use of EHR systems. Available at: http://www.americanehr.com/ research/reports/Physicians-Use-of-EHR-Systems-2014.aspx. Accessed 2014

3 Gellert GA, Ramirez R, Webster SL. The rise of the medical scribe industry: implications for the advancement of electronic health records. JAMA 2015;313(13):1315-1316

4 Shultz CG, Holmstrom HL. The use of medical scribes in health care settings: a systematic review and future directions. J Am Board Fam Med 2015;28(03):371-381

5 Ash JS, Corby S, Mohan V, et al. Safe use of the EHR by medical scribes: a qualitative study. J Am Med Inform Assoc 2020. Doi: 10.1093/jamia/ocaa199

6 Gidwani R, Nguyen C, Kofoed A, et al. Impact of scribes on physician satisfaction, patient satisfaction, and charting efficiency: a randomized controlled trial. Ann Fam Med 2017;15 (05):427-433

7 Pranaat R, Mohan V, O'Reilly M, et al. Use of simulation based on an electronic health records environment to evaluate the structure and accuracy of notes generated by medical scribes: proof-ofconcept study. JMIR Med Inform 2017;5(03):e30

8 Walker KJ, Wang A, Dunlop W, Rodda H, Ben-Meir M, Staples M. The 9-item physician documentation quality instrument (PDQI9) score is not useful in evaluating EMR (scribe) note quality in Emergency Medicine. Appl Clin Inform 2017;8(03):981-993

9 Wosik J, Fudim M, Cameron B, et al. Telehealth transformation: COVID-19 and the rise of virtual care. J Am Med Inform Assoc 2020;27(06):957-962

10 Benko S, Idarraga AJ, Bohl DD, Hamid KS. Virtual scribe services decrease documentation burden without affecting patient satisfaction: a randomized controlled trial. Foot Ankle Spec 2020: 1938640020950544

11 Brady K, Shariff A. Virtual medical scribes: making electronic medical records work for you. J Med Pract Manage 2013;29(02): 133-136

12 Corby S, Gold JA, Mohan V, et al. A sociotechnical multiple perspectives approach to the use of medical scribes: a deeper dive into the scribe-provider interaction. AMIA Annu Symp Proc 2020;2019:333-342

13 Noordzij R, Plocienniczak MJ, Brook C. Virtual scribing within otolaryngology during the COVID-19 pandemic and beyond. Am J Otolaryngol 2020;41(05):102611

14 The Joint Commission What guidelines should be followed when physicians or other licensed independent practitioners use scribes to assist with documentation?. 2020. Accessed June 8, 2020 at: https://www.jointcommission.org/standards/standard-faqs/ambulatory/record-of-care-treatment-and-services-rc/000002210/

15 Koshy S, Feustel PJ, Hong M, Kogan BA. Scribes in an ambulatory urology practice: patient and physician satisfaction. J Urol 2010; 184(01):258-262 